\title{
Long-Term, Serum-Free Cultivation of Organotypic Mouse Retina Explants with Intact Retinal Pigment Epithelium
}

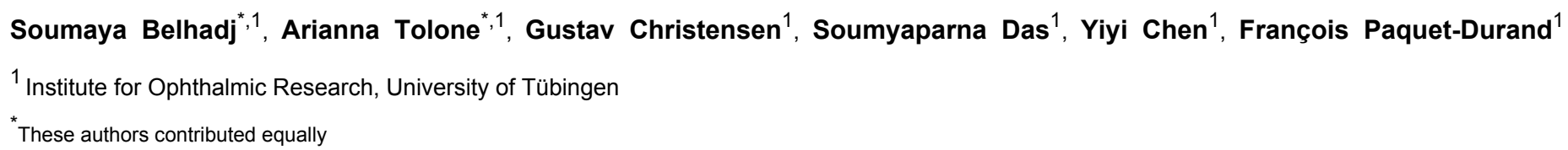

\section{Corresponding Author}

François Paquet-Durand

francois.paquet-durand@klinikum.uni-tuebinge

\section{Citation}

Belhadj, S., Tolone, A., Christensen, G., Das, S., Chen, Y., Paquet-

Durand, F. Long-Term, Serum-Free

Cultivation of Organotypic Mouse Retina Explants with Intact Retinal Pigment Epithelium. J. Vis. Exp. (165), e61868, doi:10.3791/61868 (2020).

\section{Date Published}

November 25, 2020

DOI

$10.3791 / 61868$

URL

jove.com/video/61868

\section{Abstract}

In ophthalmic research, there is a strong need for in vitro models of the neuroretina. Here, we present a detailed protocol for organotypic culturing of the mouse neuroretina with intact retinal pigment epithelium (RPE). Depending on the research question, retinas can be isolated from wild-type animals or from disease models, to study, for instance, diabetic retinopathy or hereditary retinal degeneration. Eyes from early postnatal day 2-9 animals are enucleated under aseptic conditions. They are partially digested in proteinase $\mathrm{K}$ to allow for a detachment of the choroid from the RPE. Under the stereoscope, a small incision is made in the cornea creating two edges from where the choroid and sclera can be gently peeled off from the RPE and neuroretina. The lens is then removed, and the eyecup is cut in four points to give it a four-wedged shape resembling a clover leaf. The tissue is finally transferred in a hanging drop into a cell culture insert holding a polycarbonate culturing membrane. The cultures are then maintained in R16 medium, without serum or antibiotics, under entirely defined conditions, with a medium change every second day.

The procedure described enables the isolation of the retina and the preservation of its normal physiological and histotypic context for culturing periods of at least 2 weeks. These features make organotypic retinal explant cultures an excellent model with high predictive value, for studies into retinal development, disease mechanisms, and electrophysiology, while also enabling pharmacological screening.

\section{Introduction}

In ophthalmic research, a variety of models are available to study the retina, including primary retinal cell cultures, retina-derived cell lines, retinal organoids, and in vivo animal models $^{1,2,3,4,5}$. However, each of these models suffers from drawbacks. For instance, cells grow in isolation while the retina is a complex network with a multitude of cell-tocell interactions. Thus, the behavior of isolated cell cultures is likely to be artificial compared to that observed in a 
whole tissue. This problem can in part be remedied using in vitro differentiated retinal organoids, which can be used to study development and basic biology 6 . Yet, as of today, retinal organoid generation still is time-consuming, laborintensive, and suffers from reproducibility issues, requiring substantial further development work before organoids can be used for translational retinal research. Finally, studies on live animals, while arguably the model that comes closest to the requirements of ophthalmic research, are associated with strong ethical concerns. A good compromise between the efficiency of cell culture systems and the real-life situation of in vivo animal models are organotypic retinal explant cultures. Such cultures also reduce animal suffering since no in vivo interventions are performed.

Several methods have been described for culturing retinal explants from different species ${ }^{5,7,8}$. Our protocol describes a technique for the isolation of the mouse neuroretina together with its retinal pigment epithelium (RPE). This technique will also be suitable for rat retinal cultures ${ }^{9}$. The culture of neuroretina together with its RPE is of major importance for success. The RPE performs essential functions for the retina: transport of nutrients, ions, water, absorption of light and protection against photooxidation, re-isomerization of alltrans-retinal into 11-cis-retinal, which is crucial for the visual cycle, phagocytosis of shed photoreceptor membranes, and secretion of essential factors for the structural integrity of the retina ${ }^{10}$. Maintaining the RPE allows a successful development of photoreceptor outer and inner segments, keeping the retina viable for a longer time ${ }^{11}$. The procedure described below preserves the histotypic and physiological characteristics of the retina for at least two weeks ${ }^{12}$. Moreover, culturing the organotypic retinal explants in serumfree, antibiotic-free medium avoids the presence of unknown substances and enables a straightforward interpretation of the results ${ }^{12}$.

Organotypic retinal explant cultures have been essential for improving our knowledge on retinal development and degeneration $7,13,14$. We show here that they are also a useful tool for pharmacological screening and that they can be employed to model a variety of retinal diseases, including diabetic retinopathy.

\section{Protocol}

Animal protocols compliant with $\$ 4$ of the German law of animal protection were reviewed and approved by the Tübingen University committee on animal protection (Einrichtung für Tierschutz, Tierärztlichen Dienst und Labortierkunde; Registration No. AK02/19M). In this study, retinas were obtained from wild-type (WT) and rd1 mice, the latter being a well characterized model for hereditary retinal degeneration ${ }^{15}$. Mice were housed under standard white cyclic lighting, had free access to food and water, and were used irrespective of gender.

\section{Checklist}

1. To ensure sterile conditions and avoid contaminations, clean, and disinfect the laminar air flow hood with $70 \%$ ethanol. Be sure to let the ethanol evaporate completely, to prevent intoxication of the retinal cultures.

2. Autoclave tools (e.g., scissors, forceps, and ophthalmic microscope scraping spoon) before use.

3. Prepare the following media in advance under a laminarflow hood, under sterile conditions: Basal R16 medium (BM) (can be stored at $4{ }^{\circ} \mathrm{C}$ for 4 weeks), BM with $20 \%$ fetal calf serum (FCS) (same day use), BM with $0.12 \%$ proteinase K (44 mAnson U/mg) solution (same day use) 
and complete R16 medium with supplements (CM) as described by Romijn ${ }^{16}$ (can be stored at $4{ }^{\circ} \mathrm{C}$ for 3 weeks) (see Tables S1, S2, and S3).

4. Preheat the proteinase $\mathrm{K}$ solution at $37^{\circ} \mathrm{C}$ to activate it and use it in step 2.5 .

\section{Preparation}

1. Sacrifice $r d 1 / \mathrm{WT}$ animal at post-natal day (P) 5 by decapitation. For animals older than $\mathrm{P} 11$, use $\mathrm{CO}_{2}$ and/ or cervical dislocation, as per the local animal protection regulations.

2. Depending on the age of the animal, prior to enucleation, if needed, open the eye lids using forceps and very carefully separate the eye lids, without touching or scratching the eye below.

3. Rapidly enucleate the eyes under a stereoscope using curved forceps.

4. Incubate the eyes in $\mathrm{BM}$ for $5 \mathrm{~min}$ at room temperature (RT).

5. Incubate the eyes in preheated BM, with $0.12 \%$ proteinase $\mathrm{K}$ at $37^{\circ} \mathrm{C}$ for $15 \mathrm{~min}$.

6. Perform the following steps inside a laminar air flow hood to ensure sterile conditions. To inactivate proteinase $\mathrm{K}$, transfer eyes to BM containing $20 \%$ FCS and incubate for 5 min at RT.

7. Dissect the eyes under a stereoscope, aseptically, in a Petri dish containing fresh BM at RT. Initiate the dissection as soon as possible after the enucleation. The longer this time is, the harder it is to dissect the retina, the eyes becoming very soft.

1. With forceps, hold the eye from the optic nerve. Using fine scissors, make a small incision in the cornea creating 2 edges from where the cornea, the choroid and the sclera can be gently peeled using 2 pairs of fine forceps (Figure 1 steps $A-C$ ). Alternatively, use a narrow-gauge cannula to make a first incision into the cornea and then insert one of the scissor blades into the opening.

2. Grasp the lens with fine forceps. Place a second pair of forceps perpendicularly to the first ones so that the first forceps are between the 2 shanks of the second one. Pull to extract the lens from the eye cup. If the vitreous and the ciliary body are still attached to the retina, remove them carefully (Figure 1 step D).

NOTE: Steps 2.7.1 and 2.7.2 need practice and ensure caution to not damage the retina.

3. Cut the retina perpendicular to its edges in four points, creating a four-leaf clover shape (Figure 1 steps E$\mathrm{F})$.

4. Using a pipette with broadly cut base of a $1 \mathrm{~mL}$ tip, hold the retina in a hanging drop of medium and transfer it to a culture dish filter insert placed in a 6-well culture plate. The RPE layer should face the membrane (Figure 1 step $G$ ).

5. Using a pipette, carefully remove the excess medium from the insert.

6. From the sides of the well, add $1 \mathrm{~mL}$ of $\mathrm{CM}$ per well and incubate in a sterile incubator at $37{ }^{\circ} \mathrm{C}$ with $5 \% \mathrm{CO}_{2}$. Do not submerge the retina in the medium as this will reduce oxygenation and cause tissue degeneration. The explant should remain at the interface between liquid and air, covered only by a thin film of liquid created by the surface tension of water.

8. Leave the retinal explant undisturbed for the first $48 \mathrm{~h}$ to facilitate recovery after the explantation procedure. 
9. Change the medium every second day (48 h). Discard $700 \mu \mathrm{L}$ of medium from each well and add $900 \mu \mathrm{L}$ of fresh CM to the well. In this way, the amount of medium lost by evaporation is recovered and the retinal explant keeps some of the neuroprotective factors produced in the previous $48 \mathrm{~h}$.

10. Incubate the removed medium in a separate and closed microcentrifuge tube along with the cultures to control and evaluate possible contamination (i.e., change in color of the medium).

NOTE: Retinal explants can be kept in culture for at least 2 weeks $^{12}$.

\section{After culturing}

NOTE: Explants can be used for different experimental applications (western blot, histology, whole mounts, genetic analysis, electrophysiology). Depending on the application, organotypic retinal explants can be snap frozen, lysed, or prepared for cryosectioning. The steps below describe histological preparation.

1. Perform a 45 -min fixation with $4 \%$ paraformaldehyde (PFA), followed by gradual sucrose cryoprotection $(10 \%$ sucrose for $10 \mathrm{~min}, 20 \%$ for $20 \mathrm{~min}$ and $30 \%$ for $2 \mathrm{~h}$ at room temperature $(\mathrm{RT})$ or overnight $(\mathrm{ON})$ at $\left.4{ }^{\circ} \mathrm{C}\right)$. Add these buffers directly in the well.

2. Cut the membrane around the retinal explants.

3. Embed both the membrane and retinal tissue in the medium for frozen tissue. 

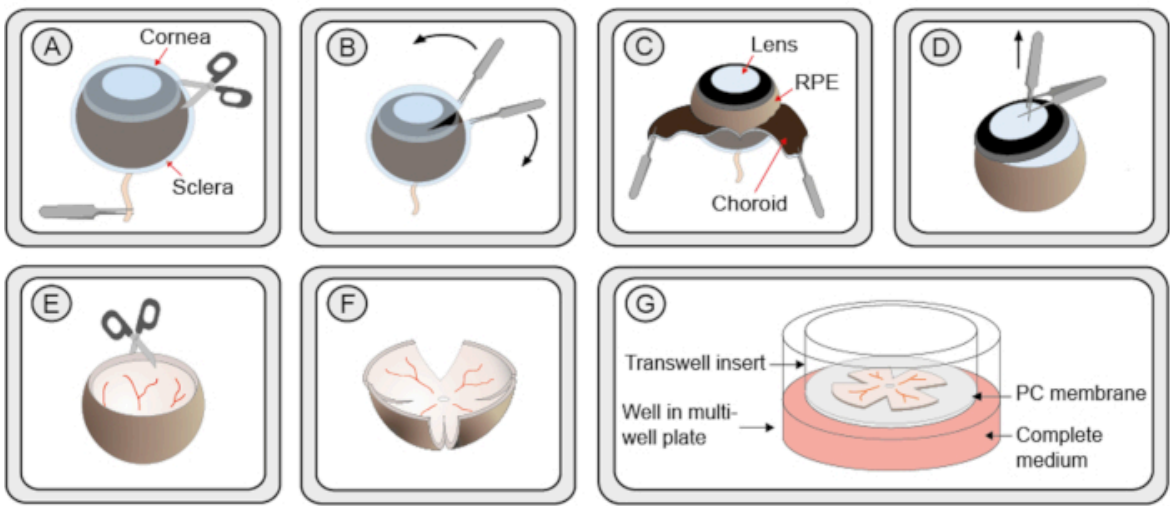

Figure 1: Step-by-step procedure for the preparation of organotypic retinal explant cultures. (A) Mouse eyes are enucleated and transferred to a solution of proteinase $\mathrm{K}$ to allow separation of sclera and choroid from the retina and RPE. A small cut in the sclera/choroid layer is introduced. (B) Two forceps are used to peel the sclera/choroid layer. (C) The black choroid layer can be seen during the peeling. The underlining dark retinal pigment epithelium (RPE) remains attached to the eyeball. The sclera and choroid are removed along with the optic nerve. (D) The lens and vitreous are extracted with forceps. Remaining ciliary body is removed. (E) The retina retains a bowl-like shape. (F) To flatten the retina for culturing in a dish, 4 cuts in equal distance around the retina are made with a scissor, giving it a clover-like shape. The retina culture is transferred to a membrane culture insert in a 6-well plate with the use of a cut $1 \mathrm{~mL}$ pipette tip. The retina still retains some of the bowl-shape. However, upon removal of the excess liquid surrounding the retina, it will unfold to a planar structure. (G) In the culture membrane setup, the retina culture is resting on a porous polycarbonate (PC) membrane on top of a solution of complete R16 medium. To ensure viability, the culture must be kept in a humidified sterile incubator at $37{ }^{\circ} \mathrm{C}$ with $5 \% \mathrm{CO}_{2}$, and the medium should be replaced every $48 \mathrm{~h}$. Please click here to view a larger version of this figure

\section{Representative Results}

After following the protocol, dissected and cultured retinal explants preserve their normal tissue architecture, with distinct layers, from the RPE to the ganglion cell layer (GCL), as shown in Figure 2. Outer nuclear layer (ONL) and inner nuclear layer (INL) size remained mostly stable for 2-3 weeks, with a slowly progressing cell loss and gradual thinning of these layers becoming more and more apparent if the culturing period is prolonged to 4 weeks and beyond. In the $\mathrm{GCL}$, in contrast, because of the axotomy of the optic nerve, a marked thinning is usually observed within the first 4 days of culturing. Afterwards, the remaining cell population in the GCL (mostly displaced amacrine cells) will continue to be viable for another 3-4-weeks ${ }^{17,18,19}$. 


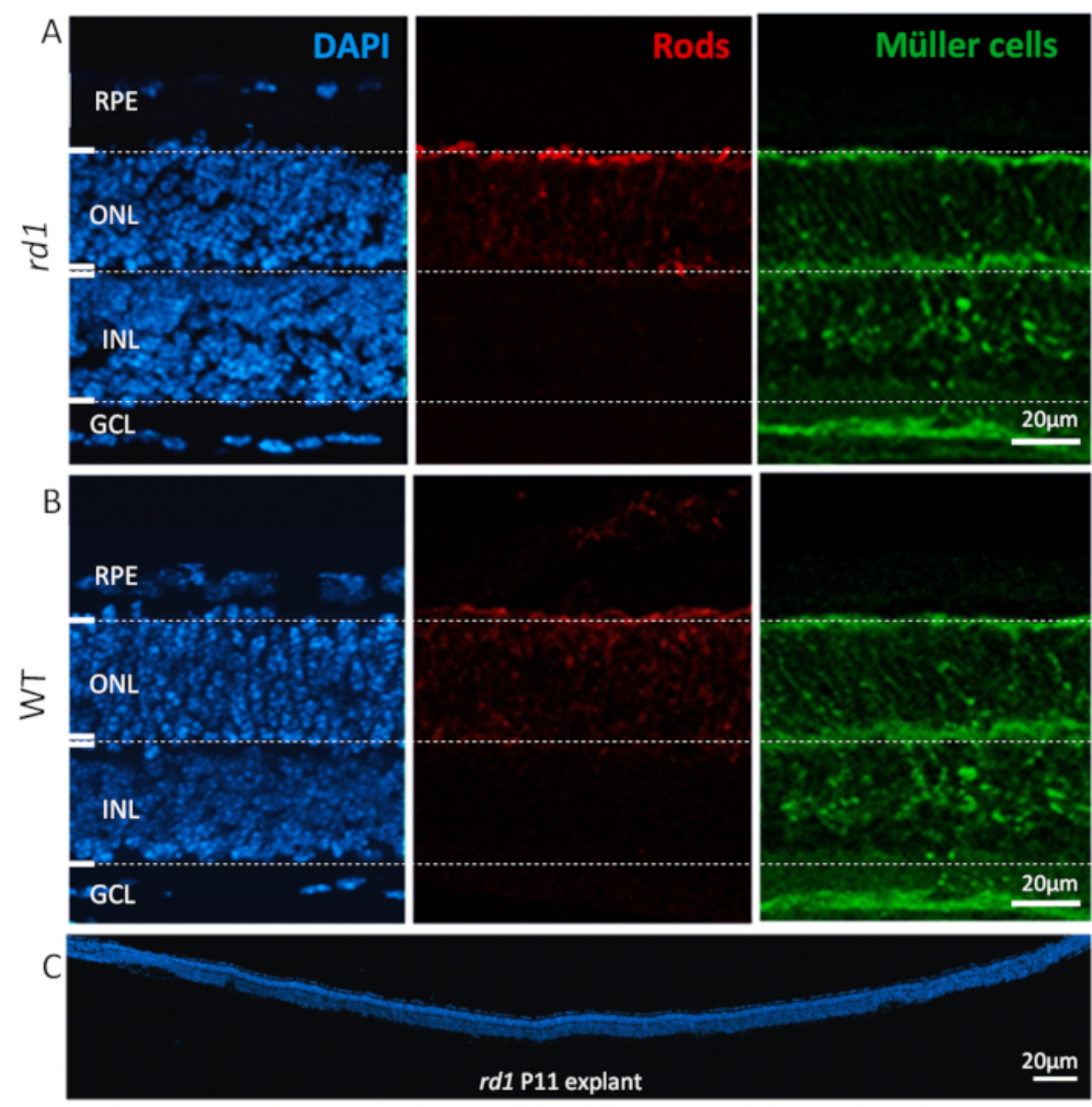

Figure 2: Cell types found in retinal explant culture. Retinal explant culture at P11 derived from rd1 mutant (A) and WT animals (B) showing nuclear staining with DAPI (left, blue), rod photoreceptors (center, red) and Müller cells (right, green). Nuclear staining highlights all the major cellular layers of the retina such as, retinal pigment epithelium (RPE), outer nuclear layer (ONL), inner nuclear layer (INL), and ganglion cell layer (GCL). Specific cell types in the nuclear layers, such as rods and Müller cells, are immunolabeled with alpha $\operatorname{arrestin}^{26}$ and glutamine synthetase ${ }^{27}$ antibodies, respectively. (C) Shows full length section of a whole rd1 mouse retina, with DAPI staining highlighting the consistency, integration, and development of the retina. These retinas were cultured for 6 days. Procedure description: Retina and RPE derived from rd1 or WT animals were isolated at P5 and cultured as described in the protocol, until P11 with a medium change every $48 \mathrm{~h}$. Cultures were fixed with 4\% PFA at P11 and cryosectioned. Please click here to view a larger version of this figure

Serum-free medium and the sustained in vitro environment allow to have full control over the experimental conditions. Here, we provide two examples for specific applications of this protocol.
The first example illustrates the possibility to use retinal explants for drug testing or screening purposes. Organotypic retinal explant cultures were prepared from wild-type (WT) and $r d 1$ mouse models. The latter is a well characterized 
model for retinal degeneration ${ }^{15}$. In the $r d 1$ mouse retina, ONL degeneration is triggered by abnormally high levels of cGMP in rod photoreceptors ${ }^{6,20}$. Excessive cGMP causes increased activity of cyclic nucleotide gated ion channels (CNGCs) and cGMP-dependent protein kinase (PKG), leading to cell death ${ }^{21}$. The treatment of $r d 1$ mouse retinas with a structural analogue to cGMP (cyclic nucleotide \#3; CN003), which targets both PKG and CNGC, was tested. After explantation at P5, the treatment paradigm described in Figure 3A was followed. Explant cultures were fixed with 4\% PFA at P11 and prepared for cryosectioning (Figure 3A). To assess cell death of histological sections from treated, non-treated (NT), and WT specimens, a terminal deoxynucleotidyl transferase dUTP nick end labeling (TUNEL) assay ${ }^{22}$ was performed. The analysis of TUNEL labeled cells showed a high percentage of dying cells in the ONL of the rd1 untreated specimens, while CN003 protected rd1 mouse photoreceptors when applied at a concentration of $50 \mu \mathrm{M}^{23}$ (Figure 3B).

A frequent complication of diabetes is diabetic retinopathy, a blinding disease which is difficult to faithfully reproduce in animal models ${ }^{5}$. The second example highlights the use of organotypic retinal explant cultures to characterize retinal cell viability under conditions emulating type-2 diabetes mellitus $(T 2 D M)^{24}$. Here, we used $20 \mathrm{mM}$ of the glycolysis inhibitor 2-deoxy-glucose (2-DG) ${ }^{25}$ and administered it to the culture medium for $24 \mathrm{~h}$ from $\mathrm{P} 10$ to $\mathrm{P} 11$. We show that subjecting WT retinal explants to such in vitro simulated diabetic conditions leads to extensive neuronal cell death of the retina (Figure $\mathbf{3 C}$ ). This paradigm in turn may then be used, for instance, to study degenerative mechanisms or to test retinoprotective treatments in a diabetes context. 


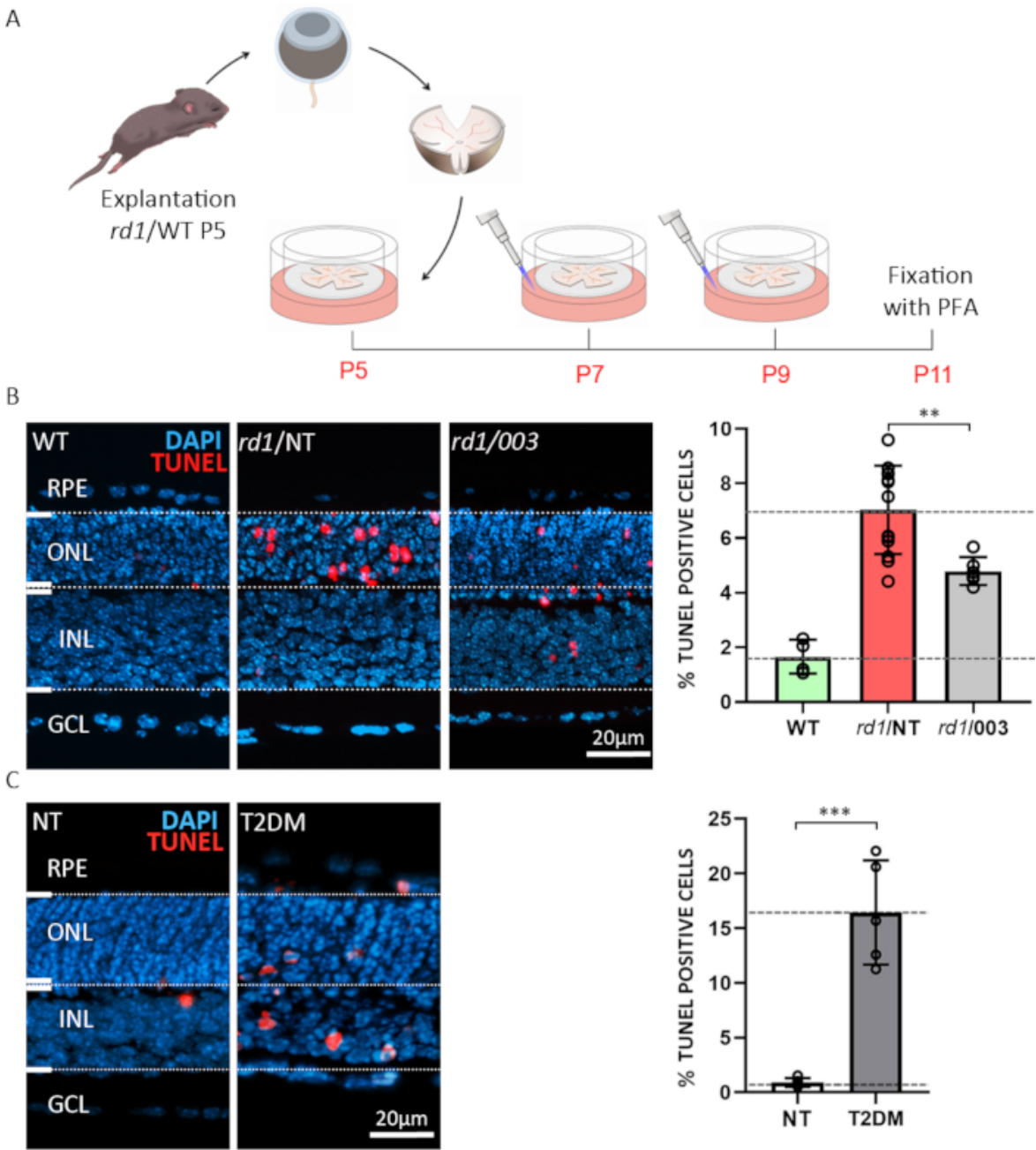

Figure 3: Two examples for applications of organotypic retinal explant cultures. (A) Procedure description: Retina and RPE derived from $r d 1$ or WT animals were isolated at post-natal day $(\mathrm{P}) 5$ and cultured as described above, with a medium change every $48 \mathrm{~h}$. At P7 and P9, the spent medium was discarded and fresh CM containing active compound at a concentration of $50 \mu \mathrm{M}$ was added to the plate. Cultures were fixed with 4\% PFA at P11 and cryosectioned. (B) Compound testing on rd1 retina. Sections were obtained from WT, treated (003), and non-treated (NT) organotypic retinal explant cultures. TUNEL assay (red) was used as a marker for cell death. Nuclear staining with DAPI (blue). The quantification shown in bar graph illustrates the percentages of dying cells in WT, rd1 NT, and rd1 003 condition. Treatment with compound 003 significantly reduced $r d 1$ photoreceptor cell death. (C) Simulation of type-2 diabetes mellitus (T2DM) on WT retina using 2-deoxy-glucose (2-DG) treatment. TUNEL assay was performed on NT and T2DM specimens. The quantification indicates a highly significant increase in cell death. Please click here to view a larger version of this figure

Table S1. Please click here to download the table

Table S2. Please click here to download the table 
Table S3. Please click here to download the table

\section{Discussion}

The protocol presented describes organotypic explant cultures of mouse retina with intact RPE in defined R16 medium, free of serum and antibiotics. This protocol was originally developed starting in the late $1980 \mathrm{~s}^{7,28}$ and since then it has been continuously refined ${ }^{6,11,12}$. Notable applications include studies into the mechanisms of hereditary retinal degeneration and the identification of retinoprotective drugs $23,29,30$

For a successful experiment, some important considerations need to be taken into account. Here are some important troubleshooting points to help enhance the quality of cultures. First, the retinal cultures may display excessive folding and/ or rosette formation ${ }^{31}$. This can be caused by touching the retina with a forceps during the explantation procedure. Moreover, the ciliary body must be completely removed from the explant, as this can increase retinal folding during culture. Second, during the transfer of the retina to the well plate in a hanging drop, if the retina faces the membrane the wrong side down, keep it in the drop hanging from the pipette tip and very gently push the medium in and out of the tip (without detaching the hanging drop) to flip the retina around. Finally, if the RPE remains attached to the sclera and detaches from the retina, it is most likely caused by an insufficient predigestion of the sclera. This problem could be especially important when working with eyes from older animals or non-rodent species (e.g., pigs) and may be resolved by increasing the proteinase $\mathrm{K}$ concentration.

Conducting organotypic retinal explant cultures is a complex procedure that requires adequate training and experience. Lack of training can lead to variability in the quality of the retinal explants. For these reasons, it is important to monitor and verify viability and reproducibility, characterizing, for instance, the rate of cell death with the TUNEL assay. The use of an antibiotic-free medium makes the retinal explants vulnerable to contamination by bacteria and fungi. To minimize this risk, we recommend that particular care is taken to work under truly aseptic conditions. Another limitation of in vitro retinal culturing are differences in physiochemical environment when compared to the in vivo retina (e.g., choroidal and retinal blood supply, oxygen and glucose levels, intraocular pressure, composition of the vitreous). A continuous perfusion system, perhaps embedded into a dedicated bioreactor ${ }^{32}$ could make this model closer to the in vivo condition. Furthermore, the axotomy of the optic nerve during retinal dissection will lead to ganglion cell death, that can induce stress responses ${ }^{8}$. Therefore, it is recommendable that the explant be left to adapt to culturing conditions for at least 2 days in vitro before it is subjected to a specific manipulation or treatment.

The described method is usually performed on immature retinal tissues, which may survive well for 4 weeks in vitro $^{7,33}$. However, the procedure is tailorable to a variety of applications, including culturing of adult retina. Although different published approaches describe the isolation of the adult retina without its $\operatorname{RPE}^{34,35}$, the incubation with papain solution for up to $1 \mathrm{~h}$ at $37{ }^{\circ} \mathrm{C}$ before dissection allows the RPE to stay attached to the retina even when derived from an adult mouse ${ }^{36}$.

The serum-free medium and the chemically defined in vitro environment provide for an entirely defined and reproducible manipulation of the experimental conditions. Therefore, organotypic retinal explant cultures are valuable tools in the field of ophthalmology and neuroscience, and have been used for studying retinal diseases ${ }^{37}$, retina 
development ${ }^{38,39}$, retinal stem cell therapy ${ }^{40}$, genetic modifications $^{41}$, and pharmacological screening. As a specific example of drug testing, here we used retinal explant cultures to test a cGMP analogue (CN003), known to reduce photoreceptor cell death in animal models for inherited retinal disease ${ }^{23}$ (Figure 3B). Another possible application of the technique is described in Figure $\mathbf{3 C}$, which illustrates how the precise control of the tissue environment can be exploited to emulate diabetic conditions ${ }^{24}$. Because of the preservation of tissue architecture over the entire culturing period, organotypic retinal explant cultures are also suitable for electrophysiological studies. Neuronal functionality on retinal explants have been investigated using patch-clamp recording 42 and multi-electrode-array (MEA) recording 33,43 . The latter allows recording of electrical activity of neuronal populations at the same time and has been exploited to characterize photoreceptor and ganglion cell functionality in culture conditions. In a broader perspective, the organotypic explant culture systems can also be applied in pre-clinical research, where explant cultures were used to test the therapeutic efficacy of hypothermia ${ }^{44}$.

The organotypic explant culturing technique is relatively simple to perform and, when compared to corresponding in vivo experiments, is less expensive and time-consuming, and avoids the ethical concerns related to live animal studies. The precise control over experimental conditions and the preservation of RPE and tissue complexity make the method a valuable tool to improve our knowledge on retinal physiology and pathophysiology and enable numerous experimental applications.

\section{Disclosures}

The authors have nothing to disclose.

\section{Acknowledgments}

This research work received financial supportfrom the European Union (transMed; H2020-MSCA-765441), the German research council (DFG; PA1751/8-1, 10-1) and the China scholarship council.

\section{References}

1. Fletcher, E. L. et al. Animal models of retinal disease. Progress in Molecular Biology and Translational Science. 100, 211-286 (2011).

2. Llonch, S., Carido, M., Ader, M. Organoid technology for retinal repair. Developmental Biology. 433 (2), 132-143 (2018).

3. Mencl, S., Trifunović, D., Zrenner, E., Paquet-Durand, F. PKG-dependent cell death in 661W cone photoreceptorlike cell cultures (experimental study). Advances in Experimental Medicine and Biology. 1074, 511-517 (2018).

4. Sanges, D., Comitato, A., Tammaro, R., Marigo, V. Apoptosis in retinal degeneration involves cross-talk between apoptosis-inducing factor (AIF) and caspase-12 and is blocked by calpain inhibitors. Proceedings of the National Academy of Sciences of the United States of America. 103 (46), 17366-17371 (2006).

5. Schnichels, S. et al. Retina in a dish: cell cultures, retinal explants and animal models for common diseases of the retina. Progress in Retinal and Eye Research. 100880 (2020).

6. Paquet-Durand, F., Hauck, S. M., van Veen, T., Ueffing, M., Ekström, P. PKG activity causes photoreceptor cell death in two retinitis pigmentosa models. Journal of Neurochemistry. 108 (3), 796-810 (2009). 
7. Caffe, A. R., Visser, H., Jansen, H. G., Sanyal, S. Histotypic differentiation of neonatal mouse retina in organ culture. Current Eye Research. 8 (10), 1083-1092 (1989).

8. Murali, A., Ramlogan-Steel, C. A., Andrzejewski, S., Steel, J. C., Layton, C. J. Retinal explant culture: A platform to investigate human neuro-retina. Clinical and Experimental Ophthalmology. 47 (2), 274-285 (2019).

9. Arango-Gonzalez, B. et al. In vivo and in vitro development of S- and M-cones in rat retina. Investigative Ophthalmology and Visual Science. 51 (10), 5320-5327 (2010).

10. Strauss, O. The retinal pigment epithelium in visual function. Physiological Reviews. 85 (3), 845-881 (2005).

11. Söderpalm, A., Szél, A., Caffé, A. R., van Veen, T. Selective development of one cone photoreceptor type in retinal organ culture. Investigative Ophthalmology and Visual Science. 35 (11), 3910-3921 (1994).

12. Caffe, A. R. et al. Mouse retina explants after longterm culture in serum free medium. Journal of Chemical Neuroanatomy. 22 (4), 263-273 (2001).

13. Caffe, A. R., Soderpalm, A. K., Holmqvist, I., van Veen, T. A combination of CNTF and BDNF rescues rd photoreceptors but changes rod differentiation in the presence of RPE in retinal explants. Investigative Ophthalmology and Visual Science. 42 (1), 275-282 (2001)

14. Ogilvie, J. M., Speck, J. D., Lett, J. M., Fleming, T. T. A reliable method for organ culture of neonatal mouse retina with long-term survival. Journal of Neuroscience Methods. 87 (1), 57-65 (1999).
15. Keeler, C. E. The inheritance of a retinal abnormality in white mice. Proceedings of the National Academy of Sciences of the United States of America. 10 (7), 329333 (1924).

16. Romijn, H. J. Development and advantages of serumfree, chemically defined nutrient media for culturing of nerve tissue. Biology of the Cell. 63 (3), 263-268 (1988).

17. Caffe, A. R. et al. Mouse retina explants after longterm culture in serum free medium. Journal of Chemical Neuroanatomy. 22 (4), 263-273 (2001).

18. Alarautalahti, V. et al. Viability of Mouse Retinal Explant Cultures Assessed by Preservation of Functionality and Morphology. Investigative Ophthalmology and Visual Science. 60 (6), 1914-1927 (2019).

19. Caffe, A. R., Visser, H., Jansen, H. G., Sanyal, S. Histotypic differentiation of neonatal mouse retina in organ culture. Current Eye Research. 8 (10), 1083-1092 (1989).

20. Farber, D. B., Lolley, R. N. Cyclic guanosine monophosphate: elevation in degenerating photoreceptor cells of the $\mathrm{C} 3 \mathrm{H}$ mouse retina. Science. 186 (4162), 449451 (1974)

21. Arango-Gonzalez, B. et al. Identification of a common non-apoptotic cell death mechanism in hereditary retinal degeneration. PloS One. 9 (11), e112142 (2014).

22. Loo, D. T. In situ detection of apoptosis by the TUNEL assay: an overview of techniques. Methods in Molecular Biology. 682, 3-13 (2011).

23. Vighi, E. et al. Combination of cGMP analogue and drug delivery system provides functional protection in hereditary retinal degeneration. Proceedings of the 
National Academy of Sciences of the United States of America. 115 (13), e2997-e3006 (2018).

24. Valdés, J. et al. Organotypic retinal explant cultures as in vitro alternative for diabetic retinopathy studies. Altex. $\mathbf{3 3}$ (4), 459-464 (2016).

25. Pajak, B. et al. 2-Deoxy-d-Glucose and its analogs: from diagnostic to therapeutic agents. International Journal of Molecular Sciences. 21 (1) (2019).

26. Slepak, V. Z., Hurley, J. B. Mechanism of light-induced translocation of arrestin and transducin in photoreceptors: interaction-restricted diffusion. IUBMB Life. 60 (1), 2-9 (2008).

27. Paquet-Durand, F. et al. A retinal model of cerebral malaria. Scientific Reports. 9 (1), 3470 (2019).

28. Romijn, H. J., de Jong, B. M., Ruijter, J. M. A procedure for culturing rat neocortex explants in a serum-free nutrient medium. Journal of Neuroscience Methods. 23 (1), 7583 (1988).

29. Azadi, S. et al. CNTF+BDNF treatment and neuroprotective pathways in the rd1 mouse retina. Brain Research. 1129 (1), 116-129 (2007).

30. Kaur, J. et al. Calpain and PARP activation during photoreceptor cell death in $\mathrm{P} 23 \mathrm{H}$ and S334ter rhodopsin mutant rats. PloS One. 6 (7), e22181 (2011).

31. Samardzija, M. et al. A mouse model for studying cone photoreceptor pathologies. Investigative Ophthalmology and Visual Science. 55 (8), 5304-5313 (2014).

32. Achberger, K. et al. Merging organoid and organ-on-achip technology to generate complex multi-layer tissue models in a human retina-on-a-chip platform. eLife. 8 (2019).
33. Alarautalahti, V. et al. Viability of Mouse Retinal Explant Cultures Assessed by Preservation of Functionality and Morphology. Investigative Ophthalmology and Visual Science. 60 (6), 1914-1927 (2019).

34. Ferrer-Martín, R. M. et al. Microglial cells in organotypic cultures of developing and adult mouse retina and their relationship with cell death. Experimental Eye Research. 121, 42-57 (2014).

35. Müller, B. Organotypic culture of adult mouse retina. Methods in Molecular Biology. 1940, 181-191 (2019).

36. Heller, J. P., Kwok, J. C., Vecino, E., Martin, K. R., Fawcett, J. W. A method for the isolation and culture of adult rat Retinal Pigment Epithelial (RPE) cells to study retinal diseases. Frontiers in Cellular Neuroscience. 9, 449 (2015).

37. Sahaboglu, A. et al. Retinitis pigmentosa: rapid neurodegeneration is governed by slow cell death mechanisms. Cell Death \& Disease. 4 (2), e488 (2013).

38. Arai, E. et al. Ablation of Kcnj10 expression in retinal explants revealed pivotal roles for Kcnj10 in the proliferation and development of Müller glia. Molecular Vision. 21, 148-159 (2015).

39. Demas, J., Eglen, S. J., Wong, R. O. Developmental loss of synchronous spontaneous activity in the mouse retina is independent of visual experience. Journal of Neuroscience. 23 (7), 2851-2860 (2003).

40. Johnson, T. V., Martin, K. R. Development and characterization of an adult retinal explant organotypic tissue culture system as an in vitro intraocular stem cell transplantation model. Investigative Ophthalmology and Visual Science. 49 (8), 3503-3512 (2008). 
41. Hatakeyama, J., Kageyama, R. Retrovirus-mediated gene transfer to retinal explants. Methods. 28 (4), 387395 (2002).

42. Moritoh, S., Tanaka, K. F., Jouhou, H., Ikenaka, K., Koizumi, A. organotypic tissue culture of adult rodent retina followed by particle-mediated acute gene transfer in vitro. PloS One. 5 (9), e12917 (2010).

43. Reinhard, K. et al. Step-by-step instructions for retina recordings with perforated multi electrode arrays. PloS One. 9 (8), e106148 (2014).

44. Klemm, P. et al. Hypothermia protects retinal ganglion cells against hypoxia-induced cell death in a retina organ culture model. Clinical and Experimental Ophthalmology. 47 (8), 1043-1054 (2019). 